\title{
Pearl millet: a sustainable cereal with low glycemic potential
}

\author{
B.D. Igbetar, C. Bosch and C. Orfila \\ School of Food Science and Nutrition, Nutrition and Public Health Group, Faculty of Mathematics and Physical \\ Sciences, University of Leeds, LS2 9JT, UK.
}

Pearl millet (Pennisetum glaucum) is a climate resilient cereal consumed widely in traditional diets in rural regions of Africa. It has a higher content of protein and minerals but is relatively lower in carbohydrates compared to maize, rice and sorghum ${ }^{(1)}$. Studies have shown potential beneficial effects of starchy food consumption on obesity, especially those containing slowly-digestible and resistant starch, and low content of sugar ${ }^{(2)}$.

In this study, the free sugar content and starch digestibility of pearl millet flour and cooked porridge were evaluated. Starch digestion was undertaken using the harmonised INFOGEST in vitro system ${ }^{(3)}$. All experiments were done in three biological replicates. Sugars were quantified using high performance anion exchange chromatography with pulsed amperometric detection (HPAEC-PAD) and results expressed as percentage yield relative to dry weight.

Our results show that free sugar content of pearl millet flour (Fig. 1A) was $2 \cdot 81 \%$, consisting mainly of sucrose and raffinose. Free sugar content increased significantly by $64.48 \%$ after cooking $(\mathrm{p}<0.05)$ suggesting endogenous amylase activity which resulted in the release of maltose and maltotriose; and invertase activity resulting in the release of glucose and fructose. Nevertheless, the total free sugar content of millet porridge is still low (less than $10 \%$ ). We observed that millet starch digestion (Fig. 1B) was rapid, reaching $44.95 \%$ and $47.63 \%$ in flour and cooked porridge respectively within 20 min. However, only $10.94 \%$ and $6.83 \%$ further digestion was observed between $20 \mathrm{~min}$ and $120 \mathrm{~min}$. Around $45 \%$ of starch was resistant to digestion after 120 min in both flour and porridge. Cooking had no significant effect $(\mathrm{p}<0.05)$ on starch digestion at any time point.

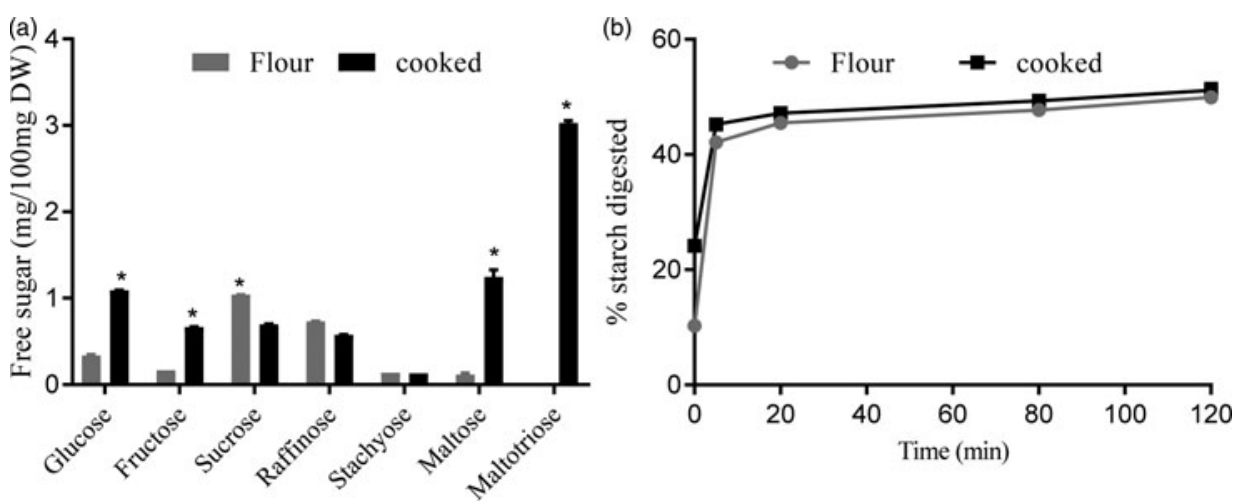

Fig. 1. Free sugar content (a) and in vitro starch digestibility (b) of millet flour and cooked porridge. Results are presented as mean of 3 biological replicates and are expressed in a dry weight basis, error bars are standard deviation of the mean. $* P<0.05$

In conclusion, high levels of resistant starch indicates low glycemic potential that needs to be verified in vivo. The consumption of millet porridge by obese populations could help them limit energy intake.

1. Saleh AS, Zhang Q, Chen J et al. (2013) Comp Rev Food Sci Food Saf 12(3), 281-295.

2. Aller EE, Abete I, Astrup A et al. (2011) Nutrients 3(3), 341-369.

3. Minekus MM, Alminger P, Alvito S et al. (2014) Food Funct 5(6), 1113-1124. 